\title{
Un personaggio in cerca di lettore: \\ Galens Großer Puls und die „Erfindung“ des Lesers*
}

\author{
Markus Asper
}

Galen ist zweifellos einer der produktivsten und einflußreichsten Fachschriftsteller der Antike, wenn nicht überhaupt der westlichen Tradition. Nicht nur als Mediziner oder als Quelle, auch als Literat verdient er unsere Aufmerksamkeit. Diese Aufmerksamkeit hat man ihm nur deshalb vorenthalten, weil gerade die einflußreichsten seiner modernen Leser es vorzogen, ihn als Vielschreiber und Polemiker zu verurteilen, ${ }^{1}$ statt diese Merkmale zu erklären (daß Kürze und Prägnanz Tugenden des Fachschriftstellers sind, war Galen natürlich bewußt). ${ }^{2}$ Im folgenden möchte ich nur einen Aspekt seiner literarischen Technik herausgreifen, nämlich die Konstruktionsarbeit, die er in die Etablierung einer AutorLeser-Bindung investiert. Dazu eignet sich das umfangreiche Handbuch (ich spreche im folgenden von „Systempragmatie“) über die Pulse aus mehreren Gründen besonders gut: erstens wird hier ein Wissen verschriftlicht, das sehr viel mit Erfahrung und Sinneswahrnehmung zu tun hat, d.h. besonders schwierig rein schriftlich zu vermitteln ist. Der Autor muß besondere Anstrengungen unternehmen, um die inhärente Unwahrscheinlichkeit der Wissensvermittlung zu minimieren, ${ }^{3}$ auch aus praktischen Gründen. ${ }^{4}$ Zweitens steht Galen hier in

* Dieser Aufsatz entstand im März 2004 am Institute for Advanced Study (Princeton, N.J.). Heinrich von Staden und Heinrich Schlange-Schöningen danke ich für Hilfe und Kritik, Thorsten Fögen für die Aufnahme in diesen Band.

Wilamowitz-Moellendorff (1886: 122 Anm. 12): „der unerträgliche seichbeutel Galen“. Galen hat geradezu hellseherisch auf Wilamowitz' Beleidigung geantwortet, und zwar sinngemäß: "Ich schreibe nicht für Deutsche (Germanen) oder andere Barbaren noch für wilde Tiere, sondern für Griechen und andere zivilisierte Völker" (San. tuend. I 10 [VI 51.8-13 Kühn]). Zu Galens „Schwächen der Darstellung“ siehe auch Deichgräber (1957: 4); ähnliche Verdikte hat Schlange-Schöningen (2003: 23-30) gesammelt.

Praes. puls. III 4 (IX 361.8 Kühn), III 5 (IX 371.4f. Kühn) und Caus. puls. II 5 (IX 74.9 Kühn). Siehe Kudlien (1972: 229 Anm. 62) mit Hinweis auf In Hippocr. Aph. XVIIIB 326f. Kühn, ferner Sluiter (1999: 186). Ansätze zur Erklärung sowohl für Vielschreiberei wie für Polemik hat Schlange-Schöningen (2003: 26-30) zusammengestellt.

Galen ist übrigens neben Platon der einzige Autor, der ein Vermittlungsrisiko bei dekontextualisierten (Fach-)Schriften als Problem erkennt. In Dign. puls. IV 2 (VIII 942.1-4 Kühn) schildert Galen einen Leser, der nichts versteht, aber „etwas Tiefes“ vermutet und „sich [sc. auf der Suche danach] zerreibt". 
einer Tradition von Pulsschriftstellern, gegen die er sich durchsetzen möchte. Das zwingt ihn dazu, darüber zu sprechen, wie gut er schreibt (bzw. wie schlecht seine Vorgänger schrieben). Drittens hat Galen zu diesem Thema mehrere Texte unterschiedlicher Formate verfaßt (siehe unten), so daß wir die große Systempragmatie stets mit anderen Formen vergleichen können. ${ }^{5}$ Ein Ergebnis dieses Vergleichs ist bereits, daß die Bereitschaft Galens, den Akt des Lesens und die persona des Lesers zu thematisieren, ein Charakteristikum dieser umfangreichen Systempragmatien ist. Viertens sind die Systempragmatien im Gegensatz zu den korrespondierenden Einführungsschriften, die meist auf Vorträgen basieren, ein Flair von Mündlichkeit bewahren und für spezielle Adressaten verfaßt sind, ${ }^{6}$ für eine vollkommen dekontextualisierte, rein schriftliche Wissensvermittlung geschrieben: ${ }^{7}$ Leser und Autor kennen einander nicht. Um so mehr Arbeit muß der Autor in den Aufbau einer Beziehung investieren, die eine erfolgreiche Wissensvermittlung ermöglicht und so sein eigenes Ansehen vergrößert, d.h. er muß eine Autorität aufbauen und versuchen, seine Beziehung zum Leser exklusiv zu gestalten.

Die Systempragmatie über die Pulse, vier mal vier zusammenhängende Bücher (De differentiis pulsuum [Diff. puls.], De dignoscendis pulsibus [Dign. puls.], De causis pulsuum [Caus. puls.], De praesagitione ex pulsibus [Praes. puls.]), bietet allein schon quantitativ einen deutlichen Kontrast zur Einführungsschrift De pulsibus ad tirones (Puls. tir.): den 40 Seiten der Einführung stehen 856 der Systempragmatie gegenüber (nach der Standardausgabe Carl Gottlob Kühns) - die Tradition spricht deshalb treffend auch vom „Großen Puls". 8 Dieses Werk verfolgt das Ziel, ohne quantifizierende Hilfsmittel wie Blutdruckmesser oder visualisierende wie Kardiogramme, sogar ohne eine exakte Zeitmessung, einen Zusammenhang zwischen Körperzuständen und Pulsvariationen darzustellen und so zu begründen, daß man den Puls als In-

4 Im Falle der medizinischen Literatur hatte der Versuch, die Vermittlungsunwahrscheinlichkeit zu bannen, auch noch einen praktischen Vorteil: hier war nämlich im Gegensatz zu anderen Disziplinen die Mobilisierung des Wissens von großer Bedeutung, da der Arzt ja mobil sein mußte und oftmals seinen Lehrer nicht erreichen konnte. Dieser mußte ihm mithin sein eigenes Wissen in einer möglichst vollkommen selbsterklärenden Form fixieren: daher der Umfang vieler galenischen Schriften. Der Versuch, Wissen ohne Einbußen mobil zu machen, führt eben nicht zu einer Reduktion von Komplexität im Text, sondern zu einer Steigerung (pace Meißner 1999: 242): die Texte müssen genauer und deshalb umfangreicher werden; sie müssen Verständnis garantieren und bauen deshalb auf Redundanz.

5 Siehe Asper (2003: 380-382, 420-430). Die Einführungsschriften Galens sind im Rahmen anderer griechischer Einführungsliteratur skizzenhaft besprochen bei Asper (1998).

6 Propr. libr. pr. (Scripta minora. Vol. 2, p. 92.25-93.1 Müller); ferner Adv. eos qui de typ. scrips. 6 (VII 507.17-508.1 Kühn). Siehe Hanson (1998: 28f.), doch vgl. Mansfeld (1994: 118f. mit Anm. 208).

7 Propr. libr. pr. (Scripta minora. Vol. 2, p. 93.4-93.15 Müller).

8 Strohmaier (1998: 266) zitiert dafür Rhazes (ar-Râzî, 865-925 n. Chr.). 
strument von Diagnose und Prognose verwenden kann. Dazu baut Galen, z.T. auf älteren Pulswissenschaftlern wie vor allem Archigenes basierend, ein umfangreiches Begriffs- und Diagnosesystem auf. Dem „Großen Puls“, der eine vollständige Wissensvermittlung beansprucht (Syn. puls. 11 [IX 463.11-24 Kühn]), kann ich hier nicht in all seinen fachliterarischen Facetten gerecht werden. Statt dessen konzentriere ich mich nur auf zwei Aspekte seiner Appellstruktur: welches Bild Galen von seinem Leser zeichnet und welches Verhältnis er zu ihm aufzubauen sucht.

\section{Appellstruktur: Der Autor und sein Leser}

Im Gegensatz zum Adressaten der Einführung kennt Galen den Leser seiner Systempragmatie nicht; entsprechend hat auch der Leser dieses Textes keine Vorstellung von Galen außerhalb derjenigen, die ihm der Text selbst vermittelt. Die Prozesse der Appellstruktur in diesem Werk lassen sich demnach als eine Doppelprojektion betrachten: Galen entwirft dem Leser ein bestimmtes Bild von sich, außerdem aber auch ein gewisses Bild vom Leser für diesen selbst. (Bei der tatsächlichen Lektüre, deren Rezeptionsvorgänge wir nicht von einem Standpunkt außerhalb des Textes überprüfen können, ist dieses Leserbild dann vermutlich eher identifikatorisch bestätigt als abgrenzend verworfen worden.)

Galens Autorentwurf besteht zunächst einmal in einer anonymen persona auctoris, die sich meist des üblichen ,integrativen Wir" bedient. ${ }^{9}$ Individualität gewinnt diese persona in der Funktion des Definierens (,Ich nenne das ... “) ${ }^{10}$ oder des Bezeugens (,I Ich habe selbst einmal gesehen, wie ..."). ${ }^{11}$ Eine Spezialfunktion und damit ein Spezialproblem solch unübersichtlicher Gebilde wie einer ausgedehnten Systempragmatie liegt im Disponieren des Stoffs. Hier bietet sich in Fällen, in denen die Wissensstruktur des Gegenstands eine Entscheidung nicht von sich aus schon nahelegt, dem Autor die Gelegenheit, sich als „disponierendes Ich“ dem Leser zu empfehlen, also bereits die Dispositionsleistung zu betonen (besonders bei Buchschlüssen). ${ }^{12}$ Das stolze „Ich des Wis-

Etwa Diff. puls. I 10 (VIII 523.5 Kühn) und I 14 (VIII 529.4f. Kühn), Caus. puls. I 8 (IX 30.10-13 Kühn) und II 13 (IX 90f. Kühn). Gelegentlich begegnet ein ,anthropologisches Wir“, z.B. in Dign. puls. I 2 (VIII 781 Kühn).

Auffällig Caus. puls. III 17 (IX 155.1f. Kühn). Unter den „authorial personae“, die von Staden für Celsus typisiert, findet sich u.a. ein „ego of dispositio“ (von Staden 1994: 110). Auch andere der personae, die von Staden herausarbeitet, finden Entsprechungen bei Galen, z.B. die "nomenclative persona“. (1994: 107; = definitorisches Ich) und das „empirical ego“ (1994: 111 ; = beglaubigendes Ich). 
senschaftlers" (je scientifique) Galen ist nicht nur von inhaltlichen Faktoren bestimmt, sondern auch von rhetorischen Strategien geprägt. ${ }^{13}$

Weiter füllt sich für einen Leser die Figur des Autors durch zahlreiche Abgrenzungsstrategien mit Persönlichkeit. Hier wird als Gegenentwurf die Individualität des Autors um so deutlicher, je genauer und persönlicher der Gegner gezeichnet wird (etwa Dign. puls. I 2 [VIII 783.16 Kühn]). Der Leser erlebt seine Galen-persona als Opfer inkompetenter Kritiker oder als kontroversenerprobten Intellektuellen, ${ }^{14}$ als einzigen Kompetenten unter den neôteroi, ${ }^{15}$ als ruhmreiche Ausnahme unter allen seinen Vorgängern, ${ }^{16}$ trotzdem nicht auf Neuerung bedacht allein um der Neuerung willen (das wäre verwerfliche kainotomia) ${ }^{17}$ Solche Abgrenzungen schaffen eine In-Group aus Autor und Leser: die anderen sind inkompetent. Dieser Prozeß unterstützt das Bedürfnis des Autors an agonalem timê-Gewinn auf Kosten seiner Mitbewerber; der Leser fühlt sich im Besitz ausgezeichneten Wissens (Schmitz 1997: 171-175). Den Leser wird es außerdem erfreuen, gerade den einzigen Autor zu lesen, der rein schriftlich das komplexe Pulswissen transportieren kann. ${ }^{18}$ Abgesehen von der Konkurrenzsituation, die derartige Selbstanpreisung erforderlich machte, könnte der Grund dafür, daß Galen seine literarischen Fähigkeiten so betont, darin liegen, daß Galens Vorgänger die Dekontextualisierung offenbar noch nicht so weit trieben, d.h. also Wissen wie die Pulslehre, das man offensichtlich am ehesten situativ vermittelt und damit praktisch lernt, gar nicht erst verschriftlichten. Galen hingegen möchte einen autonomen Text schaffen, was er dann im Anschluß klar feststellt: Dekontextualisierung heißt hier Verallgemeinerung und Fixierung (Dign. puls. I 7 [VIII 803.7-12 Kühn]) mit dem Ziel der textuellen Autonomie (autarkes). ${ }^{19}$

13 Debru (1992: 85-87) versteht die Funktion des „,je“ scientifique“ als „formule d'authenticité“, d.h. als Objektivitätssignal (das aber eben auch in einem strategischen Rahmen gesehen werden muß).

14 Diff. puls. I 1 (VIII 495 Kühn), III 2 (VIII 648.7 Kühn), IV 17 (VIII 763.15-18 Kühn).

15 Diff. puls. I 5 (VIII 508.13f. Kühn). Im ganzen Block Diff. puls. II-IV skizziert Galen sich selbst ständig als Person in Abhebung von den Pneumatikern (Kernstelle: Diff. puls. III 6 [VIII 674.9f. Kühn]).

16 Dign. puls. IV 2 (VIII 929.13-16 Kühn): Die Vorgänger haben sich fast überhaupt nicht um die Diagnose des Pulses gekümmert; ähnlich Diff. puls. I 30 (VIII 558.4 Kühn).

17 Diff. puls. II 10 (VIII $625 \mathrm{Kühn}$ ); vgl. Diff. puls. III 1 (VIII $641.13 \mathrm{Kühn).}$

18 Dign. puls. I 1 (VIII 768.9f. Kühn), I 7 (VIII 803.6f. Kühn), ferner Dign. puls. I 1 (VIII 774.3$9 \mathrm{Kühn}$ ) zum Problem, Sinneswahrnehmungen sprachlich eindeutig wiederzugeben. Galen kann das und verweist zur Illustration auf seine Weinverkostung und „-diagnose“ nach Lesewissen; das belegt aber natürlich nur, daß er eine entsprechende Lesekompetenz besitzt.

Der textuellen Autonomie, d.h. der Absicherung von Schriftkommunikation, dient auch Galens Streben nach quasi-mathematischer Argumentation (Beispiele dafür aus Plac. Hipp. Plat. bei Lloyd 1996: 259f.); daneben auch dazu, seinen eigenen Wahrheitsanspruch evident zu machen und Widersacher mit dem Hinweis auf deren logische Inkonsistenzen aus dem Felde zu schlagen. 
In diesen wenigen Zügen wird nur skizzenhaft ein Autor deutlich, dem ein naturgemäß offeneres Leserbild entspricht. Die Einführungsschrift konnte ja einen Widmungsadressaten namens Teuthras, der dem wirklichen Leser vermutlich gänzlich unbekannt war, als Identifikationsfokus verwenden. Unser Großer Puls verzichtet darauf und spricht statt dessen den Leser ständig direkt an: wie es einer dekontextualisierten Rezeption im "einsamen Akt des Lesens“ (Iser) gemäß ist, im Singular, als anonymes Leser-Du. ${ }^{20}$ Sehr selten steigert sich Galen zu an den Leser gerichteten Imperativen (z.B. Dign. puls. II 8 [VIII 861.15 Kühn]). Nach meinem Eindruck intensivieren sich diese direkten Appelle mit fortschreitender Pragmatie (um der zunehmenden Wahrscheinlichkeit vorzubeugen, daß der Leser abspringt?), gelegentlich entsprechen sich LeserDu und Autor-Ich (z.B. Dign. puls. I 8 [VIII 815 Kühn] mehrfach). Autor- und Leserkonzept stehen also in gegenseitiger, allerdings meist impliziter Abhängigkeit. Die nächste Stufe eines dekontextualisierten Leserappells, nämlich eine Wendung an eine noch weniger bestimmte dritte Person, verwendet Galen in der gesamten Pragmatie nur ein einziges $\mathrm{Mal}$, in einem hypothetischen $\mathrm{Zu}-$ sammenhang (Dign. puls. III 1 [VIII 880.5, VIII 880.16-881.1 Kühn]). Allen Leserpräfigurationen steht im Normalfall das bereits erwähnte ,integrative Wir" gegenüber und zur Seite.

Publikumsseligierende Wirkung haben Feststellungen über das Wissensniveau des Lesers, sei es negativ durch Abgrenzung zu den Adressaten der Einführungsschriften (eisagomenoi), ${ }^{21}$ sei es durch genaue Zuordnung der einzelnen Pragmatienteile zu denkbaren Lesergruppen, ${ }^{22}$ sei es durch eine Skizze

Die Beispiele sind Legion, so etwa Diff. puls. III 1 (VIII 638.5-7 Kühn), IV 2 (VIII 704.15, VIII 706.3), IV 16 (VIII 758.18-759.2, VIII 762.1 \& 8); Dign. puls. I 5 (VIII 794.13), I 6 (VIII 801.15), I 8 (VIII 810.2, VIII 811.14, VIII 812.3, VIII 819, VIII 821), II 2 (VIII 842.12, VIII 848.7, VIII 851.5f.), III 2 (VIII 889.14-19, VIII 900.11-15: Handlungsanweisung im Rezeptstil, ebenso VIII 942.12), IV 1 (VIII 917.7), IV 2 (VIII 941.17, VIII 959.7); Caus. puls. I 7 (IX 17.19, IX 18.3), I 11 (IX 46.3), II 1 (IX 57.5, IX 57.12), II 3 (IX 66.16, IX 69.10), II 12 (IX 88.2, IX 96.2).

Eisagomenoi sind gewöhnlich die Leser der Einführungsschriften. Die wenigen Ausnahmen lassen sich leicht erklären: In Diff. puls. I 5 (VIII 509.4f. Kühn) werden m.E. nicht die Adressaten des vorliegenden Buches als eisagomenoi bezeichnet; vielmehr betont Galen, daß das Gesagte für den vorliegenden Zweck bzw. Leserkreis und für eine Einführung nützlich sei und auch dort, d.h. in der Einführung, gesagt werden wird. In Dign. puls. I 6 (VIII 802.8f. Kühn) werden eisagomenoi erwähnt, die nicht identisch sind mit den eisagomenoi der Einführungsschriften; hier handelt es sich um „relative“ Anfänger, nämlich Anfänger bezogen auf die Wahrnehmung der systolê, d.h. „Leser allgemein“ (!); dazu von Staden (1998: 73 Anm. 33). Dasselbe gilt für Praes. puls. I 6 (IX 263.4-9 Kühn), wo auf eine Behandlungsdifferenz zu Diff. puls. I verwiesen wird: Dort sei der Leser noch eisagomenos gewesen, sagt Galen. Diese Ungenauigkeit werde jetzt korrigiert, weil die Leser inzwischen mehr wissen.

Dign. puls. I 1 (VIII 767.1-3 Kühn) zu den Zielgruppen der Viererteilung der Pragmatie: Diff. puls. sei für Ärzte und Philosophen gleichermaßen interessant, Dign. puls. und Praes. puls. eher für Ärzte (Diagnose und Prognose), Caus. puls. eher für Philosophen. 
erforderlichen Vorwissens (z.B. Praes. puls. I 1 [IX 205.3-6 Kühn]). Wer auf dem Weg zur völligen Beherrschung des Stoffes ist, liest Galen (Praes. puls. I 1 [IX 206.8 Kühn])! So darf der Leser auch den Umkehrschluß wagen: er liest Galen, ist also auf bestem Wege. Publikumsselektion, Sympathielenkung und Leserbindung gehen hier eine sehr enge Bindung ein. Daß Galen daran liegt, den Leser an sich als einzige oder doch wichtigste Wissensquelle zu binden, zeigt bereits die Fülle der Verweise und der Hinweis auf die Bedingtheit der Schlüsse in vorliegenden Schriften, die regelmäßig von Voraussetzungen in anderen Schriften abhängen. Galen zu verstehen ist „nicht schwer“, wie er sagt, „wenn man bisher alles von mir genau gelesen hat" (Diff. puls. I 18 [VIII 545.11-13 Kühn]). Galen geht es ganz offensichtlich um eine Monopolisierung seines Publikums (siehe unten).

Galens extremste und gleichzeitig literarisch ambitionierteste Maßnahme der Leserbeeinflussung ist der lebhaft gezeichnete Entwurf des Ideallesers. Eingeleitet von einer Skizze des Anti-Lesers, des spitzfindigen "Sophisten“ (cf. von Staden 1997: 34-36) stellt sich Galen seinen Leser als einen Liebhaber der Wahrheit vor - sollte es auch nur einer sein unter Zehntausenden, sollte dieser eine auch erst geboren werden. ${ }^{23}$ Diese „Fiktion vom dem einen würdigen Leser" ${ }^{24}$ dient rein rhetorischen Zwecken und ist als eine schlichte Werbemaßnahme zu verstehen, die jedem Leser die Sicherheit verkauft, zu den wenigen Auserwählten zu gehören. Erlös ist die timê des Autors. Da Galen sich selbst oft als Leser der Schriften seiner Debattengegner schildert, wird aus solchen Scheindiskussionen noch zusätzlich eine negative Differenz zum Leseerlebnis seiner Leser deutlich. Denn all die literarischen Fehler seiner Schriftstellerkonkurrenten begeht er selbst natürlich nicht! Die polemisch Abgekanzelten übernehmen also die Funktion von Differenzautoren, ${ }^{25}$ vor deren Versagen Galen sich um so strahlender abhebt.

Es hat wenig Sinn, aus derartigen literarischen Strategien Schlüsse auf Galens Charakter zu ziehen: Diese elaborierten Techniken der Leserlenkung sind vielmehr als notwendige Folgen einer Ausweitung der ärztlichen Konkurrenz auf das Feld der Texte, als Reaktion auf die steigende Komplexität des Wissens und auf den mit der zunehmenden Verbreitung schriftlicher Wissensvermittlung steigenden Dekontextualisierungsgrad ihrer Medien zu verstehen.

23 Dign. puls. I 8 (VIII 808.6-19, bes. 12-15), II 1 (VIII 826.17-827.3 Kühn), II 2 (VIII 859.14$860.3 \mathrm{Kühn})$.

24 So Deichgräber (1957: 26 Anm. 1), der richtig bemerkt, diese Figur habe „etwas Epideiktisches" (Deichgräber 1957: 29).

25 Sehr deutlich etwa Archigenes in Dign. puls. IV 2 (VIII 941.17f. Kühn). 


\section{Die „Erfindung“ des medizinischen Lesepublikums}

Das schriftliche Werk stellt eine Transposition der alten, mündlichperformativen epideixis in ein neues Medium dar. Das Lesepublikum, der neue Adressat, ${ }^{26}$ ist eine Erweiterung des Publikums ursprünglicher mündlicher Kommunikation, sei es im Ausbildungsverhältnis der apprenticeship oder als Adressat einer epideixis. ${ }^{27}$ Über die bisher aufgeführten Facetten der Appellstruktur hinaus führen zwei Komplexe, die zusammengenommen eine neue Leserrolle beschreiben, also quasi ein Lesepublikum erfinden. Das ist erstens eine neue Bestimmung des Verhältnisses von Lesen und Praxis und zweitens die Vorstellung, Lesen sei eine erweiterte apprenticeship.

Das gerade in medizinischer Literatur stets prekäre Verhältnis von Praxis und Lektüre scheint von zahlreichen Beteuerungen Galens eindeutig zugunsten der Praxis bestimmt zu werden: Wer nicht in praktischer Unterweisung, d.h. mündlich und situationsgebunden, von einem Lehrer, gelernt habe, der sei wie ein Steuermann, der aus Büchern navigiere; so oder ähnlich äußert sich Galen häufig. ${ }^{28}$ Wie verträgt sich diese Haltung aber mit seinem ausgedehnten Schreiben? Für seine eigenen Schriften nimmt Galen nun eine Ausnahme von der gängigen Antithese von Theorie und Praxis in Anspruch: Er preist seine Schriften dem Leser ganz einfach nicht als Gegensatz zu praktischer Lehre an, sondern als ihre konsequente Fortführung oder teilweise sogar Kompensation. ${ }^{29}$ Galens Schriften seien, so ihr Autor, aufgrund ihrer hohen fachlichen wie besonders ihrer schriftstellerischen Qualität in der Lage, den praktischen Wissenserwerb zu systematisieren, wenn man sie nämlich vorher lese, ${ }^{30}$ und damit erheblich zu verkürzen (jedoch nicht: die Praxis vollkommen überflüssig zu machen). ${ }^{31}$

Im Gegenzug zu dieser Aufwertung des Vermittlungswegs über das Buch gleicht Galen die Leserezeption der harten Arbeit der Praxis an: er skizziert Lesen als Mühe, sein idealer Leser ist ein Arbeitstier - aber eines, dessen Re-

26

27

Z.B. hoi anagnôsomenoi in Propr. libr. pr. (Scripta minora. Vol. 2, p. 90.11 f. Müller). Siehe Drabkin (1944: 350) und Temkin (1953: 215).

Siehe z.B. De anat. admin. (arab. 11.11, p. 131 Simon; Übersetzung von W. L. H. Duckworth, Cambridge 1962, 104f.): Galen verfaßt das Werk auch für jene, die seinen anatomischen Demonstrationen nicht persönlich beiwohnen konnten.

Siehe etwa Propr. libr. 5 (Scripta minora. Vol. 2, p. 110.25-27 Müller), De simpl. med. temp. ac fac. VI pr. (XI 796f. Kühn), De comp. med. sec. loc. VI 1 (XII 894 Kühn). Zur Vorgeschichte des Vergleichs Platon, Polit. 297e-299e; Polybios, Hist. 12.25d.5f. Zum Problem siehe Meißner (1999: 230-234), der aber Galens Äußerungen zu wenig hinterfragt.

Dign. puls. I 1 (VIII 773.2-6 und 14f. Kühn); siehe auch Praes. puls. IV 8 (IX 405.6 Kühn).

Siehe Puls. tir. 12 (VIII 478.5f. Kühn): Abkürzung des langen Arbeitsprozesses. Morb. temp. 7 (VII 430.9-14 Kühn): Die Diagnose einer speziellen systolê bedarf einer Übung, für die Galen-Lektüre Bedingung ist.

So ausführlich die Pulspragmatie auch ist: niemand kann ausschließlich aus ihr das Metier lernen. Wenskus (1997) hält das für Kalkül; eher liegt es an der Art des Wissens. 
vier der Schreibtisch ist (Dign. puls. I 1 [VIII 773.13-15 Kühn]). So wird Lesen Forschung, Theorie wird Praxis. ${ }^{32}$ Solche Bemerkungen sind vor dem Hintergrund zu verstehen, daß die Patienten im allgemeinen vermutlich Ärzte mit praktischer Erfahrung Theoretikern vorgezogen haben (vgl. Lukian, Hipp. 1) und daß Galen von seinen Konkurrenten dementsprechend als „Wortarzt“ (logiatros) denunziert wurde. ${ }^{33} \mathrm{Um}$ diesen Vorwurf abzuwehren und dennoch grosse Kreise mit Schriften ansprechen zu können, mußte Galen eben den Gegensatz von Theorie und Praxis aufheben. Schreiben und Lesen werden so zu Praktiken eigenen Rechts, die medizinischer Praxis ähnlich und damit gleichwertig sind.

Diesen Schluß stützt Galens offensichtliche Auffassung seiner Weise der dekontextualisierten Wissensvermittlung durch Bücher als einer erweiterten Form der alten apprenticeship. Die Wissensvermittlung der Medizin, die sich nach Galen aus einer rein familieninternen apprenticeship unter den Asklepiaden durch schrittweise Vergrößerung des Adressatenkreises immer mehr dekontextualisierte und irgendwann verschriftlichte (tendenziell trifft Galen damit wohl das Richtige), beschreibt er selbst als Degeneration: denn immer schlechter sei die technê dadurch geworden - was Mediziner mit bestimmten Schriften zu kompensieren versuchen. ${ }^{34}$ Galens Ziel ist es, wenn möglich alle Menschen zu belehren, und zwar so, wie man selbstverständlich ergänzt, daß die technê eben nicht immer schlechter werde. Daß ein produktiver medizinischer Schriftsteller wie Galen einerseits den Vorrang direkter mündlicher Wissensvermittlung betont, im selben Atemzug aber die eigenen Abhandlungen empfiehlt, weil sie im Gegensatz zu den angeblich dürren Abrissen der Kollegen die Nachteile der Schriftlichkeit durch eine ausführlichere Darstellung kompensierten, ${ }^{35}$ zeigt deutlich diesen Übergangscharakter von einem mündlich zu einem schriftlich dominierten System der Wissensvermittlung. Mit der Einsicht Galens in die Geschichte medizinischer Wissensvermittlung kommt die angebliche Degeneration also an ihr Ende, weil Galen mehr Wissen verschriftlicht und dies noch dazu besser als seine Vorgänger. ${ }^{36}$

32 Caus. puls. I 1 (IX 3.16f. Kühn). Siehe auch Temkin (1973: 33f.).

33 Propr. libr. 1 (Scripta minora. Vol. 2, p. 96.11 Müller): Diese (sicher waren es Praktiker) bezeichnet Galen dann seinerseits als antitechnoi. Vielleicht spielt hier auch die Konkurrenz von römischen und griechischen Ärzten (aus griechischer Sicht gewiß antitechnoi) eine Rolle (Nijhuis 1995: bes. 57-59).

34 Anat. adm. II 1 (II 280.2-283.3 Kühn); dazu Meißner (1997: 58). Schriftlichkeit verbunden mit Degeneration in der Medizin findet sich auch bei Celsus (von Staden 1999: 261-264).

35 So De alim. fac. I 1 (VI $480.8 \mathrm{f}$. Kühn); vergleichbar De simpl. med. temp. ac fac. VI pr. (XI 797 Kühn); De comp. med. sec. loc. VI 1 (XII 894 Kühn). Siehe van der Eijk (1997: 96 Anm. 68).

36 Siehe Dign. puls. I 7 (VIII 803.6-12 Kühn). Ähnlich wird ein Lesepublikum deutlich bei Heron, Belop. p. 18 Marsden: seine Vorgänger haben nicht für Dekontextualisierung geschrieben, wie er sagt. 
Nun hat Galen aber offenbar gar keine Alternative mehr zu einem Lesepublikum gesehen: Er war selbst in keinen institutionellen Schulkontext eingespannt, ${ }^{37}$,Institution“ hier im lockeren Sinne der philosophischen oder medizinischen hairesis verstanden. ${ }^{38} \mathrm{Er}$ hat außerdem auch selbst keine Schule gegründet. ${ }^{39}$ So kann er auch die schulgebundenen Medien, vor allem ihre charakteristische Zweiteilung in „exoterische“ und „esoterische“ Schriften nicht für sich akzeptieren. ${ }^{40}$ Dementsprechend legt er bei der Bewertung von Medizinern auch keinen Wert auf deren akademische Herkunft: bei der Ärzteprüfung in De optimo medico cognoscendo spielt keine Rolle, woher, d.h. aus welcher Schule, der Arzt kommt, wo er studiert hat usw. (so auch Nutton 1990: 248). Größten Wert aber legt Galen darauf festzustellen, ob der zu bewertende Arzt liest. ${ }^{41}$ Das könnte man daraus erklären, daß Galen selbst seinen Einfluß eben über Schriften gesucht hat, d.h. die Konkurrenz um timê konsequent auf ein neues Feld ausweitete. Die öffentliche Konkurrenzsituation der epideixis findet nun im Buch statt; der Rezipient nimmt sein Wissen aus Galens Schriften, wie im Goldenen Zeitalter der Medizin der Sohn vom Vater lernte. Hier läßt sich ein regelrechter Medienwechsel feststellen.

Ein weiterer Aspekt dieses Medienwechsels ist die briefliche Konsultation, deren Galen sich rühmt. ${ }^{42}$ Er hat also nicht nur die medizinische Wissensvermittlung mit dem hochdifferenzierten Spektrum seiner Schriften weitergehend dekontextualisiert, als es gemeinhin üblich war, sondern auch die Praxis des Arztes in diesen Differenzierungsproze $\beta$ miteinbezogen ${ }^{43}$ Eine weitere dieser Ausweitungstendenzen erkenne ich in der gerade erwähnten Schrift De optimo medico cognoscendo. Bei dieser Schrift handelt es sich um einen Versuch Galens, ein neues Publikum für seine eigenen Schriften zu erschließen, gewissermaßen seinen Markt zu erweitern. Neben den professionellen Lesern sollten

Dazu Kudlien (1972: 233), Temkin (1973: 36), Mansfeld (1994: 124f.) und Horstmanshoff (1995: 87).

Eine feste Institutionalisierung der in Rom tätigen Ärzte scheint es vor dem 4. Jh. n. Chr. nicht gegeben zu haben (Nutton 1971: 62).

Was manche Historiker generell gewundert hat, z.B. Scarborough (1981: 2, 22). Doch spricht Galen gelegentlich von hetairoi und deren Schülern (Comp. med. per gen. III 2 [XIII 603.10$12 \mathrm{Kühn}$ ], De semine II 1 [IV 595.10f. Kühn = CMG V 3.1 p. 146.11]; siehe auch Mansfeld (1994: 124), sah sich also doch in eine irgendwie geartete Gruppe eingebettet.

40 Zur Kritik Galens an der Unterscheidung von „exoterischer" und „esoterischer" Literatur siehe Plac. Hipp. Plat. III 4.12f. (= CMG V 4.1.2 p. 194.16-27); siehe Hanson (1998: 30).

41 Siehe z.B. Opt. med. cogn. p. 69.15f., 97.9-12 Iskandar. Paradigmatisch für die neue Rolle des Lesens ist das Auftreten des Philologen als Schiedsrichters der Ärzte in De praecogn. 5 (XIV 630.9-14 Kühn [= CMG V 8.1 p. 100.2-6]).

In De loc. aff. IV 2 (VIII 224.8f. Kühn) führt er Iberia, Keltike, Asien und Thrakien an. Dazu Nutton (1984: 316) und Horstmanshoff (1995: 86). veröffentlicht (zitiert von Galen, Loc. aff. III 5 [VIII 150.5 Kühn]); dazu Wellmann (1895: 21 f. mit Anm. 10), 
Angehörige der römischen Oberschicht mit einem Interesse an Medizin hinzukommen. ${ }^{44}$ Ich halte es für wahrscheinlich, daß es sich bei diesem Kreis um eine medienspezifische Erweiterung des Publikums der epideixis handelte. Legt man die aristotelische Typologie der medizinisch Wissenden zugrunde, ${ }^{45}$ so zielt Galen offenbar neben den Praktikern ${ }^{46}$ gelegentlich auch auf medizinisch interessierte, wohlhabende Laien (philiatroi). ${ }^{47}$ Diese Gruppe kann nicht groß gewesen sein. Entsprechend intensiv dürften die Ärzte um sie konkurriert haben. Mit seinem Streben nach dieser Ausweitung des Publikums war Galen erfolgreich: Medizinschriftsteller werden zu beliebten Autoren der upper class (zu Oreibasios siehe Scarborough 1981: 22). Alle diese publikumsbildenden Maßnahmen und publikumsbindenden Strategien fasse ich als „Erfindung“ eines Lesepublikums zusammen. Daß Galen das Lesen und die Problematik einer schriftlichen Wissensvermittlung bewußt war und am Herzen lag, beweisen seine Essays zu theoretischen Aspekten einzelner Situationen der Wissensvermittlung (zur epideixis, zum dialogos) und zu Leseverhalten sowie Hörerrezeption. $^{48}$

Vermutlich ist diese „Erfindung“ des Lesepublikums im Kontext der Zweiten Sophistik zu sehen, einer generellen Ausweitung von Lesekommunikation mit allen möglichen Wirkungen: Verlagerung oder Differenzierung von Agonistik auf den Leseakt, Ausweitung der Schriftproduktion, Verlagerung von bisher mündlichen Formen der Wissensvermittlung auf das neue Medium, Kanonisierung. ${ }^{49}$ Daß Galens Leser außerhalb jeder Institutionalisierung steht, erfordert ja gerade erst die oben geschilderten Maßnahmen. Der Leser ist nur noch im Text zu erreichen. Der Leseprozeß muß neu determiniert werden, weil bestimmte Rezeptionshilfen, die in der Regel von den wissensvermittelnden Institutionen vorgegeben werden, jetzt wegfallen. Natürlich soll hier nicht behauptet werden, daß Galen überhaupt auf mündliche Wissensvermittlung verzichtete (er spricht ja oft genug von seinen epideixeis). ${ }^{50}$ Doch dürfte bei diesen

44 Dazu Nutton (1990: 244), Horstmanshoff (1995: 88-90) und Schlange-Schöningen (2003: 167f.). Allgemein zu „Bildung“ als Distinktionswissen der lokalen Eliten im 2. Jh. n. Chr. Schmitz (1997: 101-110).

Pol. III 11 1282a3-5: Praktiker (dêmiurgos), Theoretiker (architektonikos) und gebildeter Laie (ho peri tên technên pepaideumenos); dazu Kudlien (1985: passim).

46 Diese hatten zunehmend theoretische Aspekte zu bewältigen; siehe dazu die von Kollesch (1979: 512) erwähnte Approbation unter Septimius Severus.

47 Horstmanshoff (1995: 89f.) mit Verweis auf San. tuend. I 10 (VI 50 Kühn); vgl. Bowersock (1969: 71-74).

48 Zwei kurze Schriften erwähnt er in Propr. libr. 12 (Scripta minora. Vol. 2, p. 121.12-14 Müller): Peri tês tôn epideiknymenôn 〈pros〉 tous akouontas synousias, Peri tôn anagignôskontôn lathra (der letzte Titel ist anders gedeutet bei Mansfeld 1994: 127). 53).

50 Siehe Ilberg (1974: 18f.) zu Galens anatomischen Schriften. 
Veranstaltungen die Mehrung und Vermittlung von Reputation wichtiger gewesen sein als die Wissensvermittlung. Insgesamt kehrt Galen die Gewichtung um: Sein schriftliches Opus besteht nicht mehr nur aus den verschriftlichten Vorträgen und thesaurierenden Materialsammlungen, sondern vor allem aus konzeptuell schriftlichen Monographien und Systempragmatien, in denen das nunmehr autonome Wissen auf seinen unbekannten Leser wartet.

\section{Anxiety of influence: Die Monopolisierung des Lesers}

Dieser unbekannte Leser muß nun aus der Sicht Galens vor allem vor den Schriften der anderen geschützt werden: Galen bringt es demgemäß fertig, im selben Atemzug gut platonisch zu behaupten, die Leserezeption sei im Grunde dem Anhören einer lebendigen Vorlesung unterlegen, und daraus auch noch ein Argument für die Lektüre seiner eigenen Schriften zu drechseln: Obwohl er nämlich mehrfach betont, daß die effizienteste Unterrichtsweise sich „lebendiger Stimme" bediene (natürlich meint er seine eigene) und daß allein aus Büchern noch keiner zum Experten geworden sei, ${ }^{51}$ zögert er doch nicht, seine eigenen Schriften denjenigen zu empfehlen, die gerade keinen Zugang zu Lehrern haben. Seine eigenen Lehrbücher nimmt er vom allgemeinen Verdikt offenbar deshalb aus, weil sie auf die Situation reiner Leserezeption durch konzeptionelle Schriftlichkeit (gegrammena im impliziten Gegensatz zu hypomnêmata) und durch eine kompensatorische Steigerung des methodischen Aufbaus (saphôs te kai kata diexodon) Rücksicht nehmen und deshalb gerade keine hypomnêmata sind, die nur Experten daran erinnern können, was sie einst gelernt haben, aber keine primäre Wissensvermittlung zustandebringen (De alim. fac. I 1 [VI 480 Kühn]). Dieses Problem erwähnt bereits Aristoteles, der medizinische Fachliteratur demnach auch nur für fachinterne Kommunikation zuläßt. ${ }^{52}$ Aristoteles spricht offensichtlich für eine Zeit, die noch keine Einführungsschriften, überhaupt keine vollständig dekontextualisierte und nach Funktionen differenzierte Schriftkommunikation kennt.

$\mathrm{Da}$ sein Leser nicht in falsche Hände gerät, sondern sich allein mit Galen als dem väterlich sorgenden, ${ }^{53}$ d.h. monopolisierenden, Autor befaßt, dafür sorgen die typisch galenischen Maßnahmen der Autokanonisierung und Autotextpflege, die etablierte Praktiken und Gattungen der Klassikerphilologie auf das eigene Euvre übertragen (Mansfeld 1994: 3-5). Die markanteste dieser philologischen Praktiken ist die Regelung der Lesereihenfolge (taxis) in zahl-

\footnotetext{
51 Zu diesem Topos siehe Mansfeld (1994: 123) mit Verweis auf Seneca, Epist. 6.5f. (viva vox); jetzt auch Sluiter (2000: 191). EN X $9118 \mathrm{lb2}-6$ : Fachbücher sind toîs men empeirois ôphelima [...], tois d' anepistêmosin achreia.

53

Zur Wissensvermittlung vom Vater an den Sohn siehe z.B. Althoff (1993: 216f.)
} 
reichen Nebenbemerkungen ${ }^{54}$ und in eigenen Werken (Ord. libr. propr.). All das dient dem Ausgleich eines Defizits an Kontextwissen, d.h. also der Kompensation einer Unterdetermination von Lektüre, die aus Dekontextualisierung entsteht. ${ }^{55}$ Galen tut einfach alles, um sich selbst zum Klassiker zu machen, oder: er „erfindet“ auch noch sein künftiges Lesepublikum. ${ }^{56}$ Wie gut das funktionierte, zeigt sein Nachruhm, den er viel mehr als Medizinschriftsteller genoß, weniger als Arzt: Charakteristisch ist die Erwähnung bei Athenaios, für den die Reputation Galens vor allem auf seinen Schriften und nicht zuletzt deren Quantität beruhte, die ihn in die Nähe der Alten rücke. ${ }^{57}$ Der Erfolg Galens drückt sich im Ehrentitel "medical dictator" aus, der Galen von modernen Medizingeschichtlern gelegentlich beigelegt wurde. ${ }^{58}$

Galens Begierde, sich ein Publikum zu erobern, der sich all die geschilderten Züge der Systempragmatie und viele der anderen Schriften unterordnen, ist vermutlich ein Ausfluß der allgemeinen Distinktionsbemühungen der Oberschicht seiner Zeit. Ins Literarhistorische transponiert läßt es sich mit Harold Bloom als , anxiety of influence“ bezeichnen, die „starke“ Autoren kennzeichne (Bloom 1973: 5).$^{59}$ Galen geht es um die Usurpation, die Monopolisierung einer Tradition. ${ }^{60}$ Damit war er über alle Maßen erfolgreich (Nutton 2002: 251f.). Seine „Einflußangst“ zeigt sich konzeptgemäß im Umgang mit seinen Vorgängern, in der Pulspragmatie besonders mit dem renommierten Arzt Archigenes: Galen hängt wahrscheinlich selbst zwar weitgehend von Archigenes $a b,{ }^{61}$ greift aber gerade ihn erbarmungslos an, demontiert also seine Hauptquel-

54 Z.B. Dign. puls. I 2 (VIII 780.8-14 Kühn), Syn. puls. 11 (IX 463.6-14 Kühn); siehe Gossen (1907: 28).

55 Dasselbe gilt für Libr. propr. und Plac. propr. Diese Schriften arbeiten wie die ständigen Wiederholungen und die zahllosen Querverweise im Corpus Galenicum am enzyklopädischen Charakter des Gesamtwerks (Vegetti 1994: 336f.). Parallelen zu Augustinus' Retractationes und Porphyrios' Vita Plotini entdeckt Mansfeld (1994: 117f. mit Anm. 206).

56 So lassen sich Ord. propr. libr. und Propr. libr. auch als „Vermächtnis“ verstehen (Mansfeld 1994: 125).

57 Der Tenor ist „Galen hat mehr Bücher veröffentlicht zu Philosophie und Medizin als alle seine Vorgänger". Siehe vor allem Deipn. 1, 1e, außerdem 26c-27d und 3.115c-116a (dazu Temkin 1973: 59f.; Nutton 1984: 317).

58 Zuerst von Cowlishaw (1937). Wie übrigens Meth. med. II 5 (X 105.13-106.1 Kühn) zeigt, hätte Galen das Prädikat „Diktator“ oder „Tyrann“ abgelehnt (doch vgl. Anm. 60).

59 Von den sechs Techniken dieser anxiety, die Bloom (1973: 14-16) schildert, treffen alle bis auf die vierte auf Galen zu.

60 Z.B. De opt. doctr. (CMG V 1.1 p. 104.18): Der Adressat soll keiner anderen didaskalia bedürfen als derjenigen Galens. Richtig Sarton (1954: 49): „Galen was primarily a writer, who craved philosophical and literary fame" (Kursive von mir). Aussagen Galens, er habe sich nie um persönlichen Ruhm gekümmert, ja ihn sogar zu verhindern versucht (etwa Meth. med. VII 1 [X 456.5-7, X 457.15-17 Kühn]), dienen lediglich der Selbstinszenierung.

61 Caus. puls. I 7 (IX 18.18 Kühn): Magnos wird aus Archigenes zitiert. Wellmann (1895: 170201) hat gezeigt, daß Galen in seinem Kategoriensystem fast vollständig auf Archigenes' Peri sphygmou beruht. 
le systematisch. Dasselbe läßt sich auch in anderen Schriften beobachten. ${ }^{62} \mathrm{Mit}$ diesem Vorgehen hat Galen seine Vorgänger, also die Konkurrenten um die Gunst gegenwärtiger und zukünftiger Leser, erfolgreich $\mathrm{zu}$ verdrängen versucht. ${ }^{63}$ Anxiety of influence als eine grundsätzliche Form der Kommunikation zwischen Autor und Leser ist nun meines Erachtens zu verstehen als eine Radikalisierung des archaischen (und noch kaiserzeitlichen) Agonalitätsprinzips durch Schrift und gleichzeitig als Auffassung einer Wissensvermittlung an Leser als apprenticeship, mit der eifersüchtig eine exklusive Bindung des Adepten an den Lehrer gesucht wird. ${ }^{64}$ Diese Eifersucht liegt in der Dekontextualisierung von Wissen durch die Schrift begründet: der Wettbewerb von Autoren radikalisiert sich, indem er sich auf verschiedene Zeiten und verschiedene Orte ausdehnt. Es erhebt sich ein Agon aller gegen alle: als Schriftsteller muß Galen nicht nur gegen die einschlägigen Klassiker und seine Zeitgenossen bestehen, sondern sogar gegen die, die nach ihm kommen werden. Anxiety of influence ist eben die resultierende Gier des Autors nach unbedingter und ausschließlicher Leserbindung und entsprechender timê auf Kosten seiner Konkurrenten und besonders seiner Vorgänger.

Die stärkere Hinwendung Galens zu einer Leserezeption ist auch als Folge oder wenigstens im Rahmen der Theoretisierung oder Philologisierung der Medizin seit dem Hellenismus zu sehen, die sich im Umbruch zu einer zunehmend textbasierten Wissensvermittlung befand. ${ }^{65}$ Schon der Empiriker Archibios hatte etwa ein halbes Jahrhundert vor Galen gegen ein zu wenig „empirisches", zu textlastiges Studium argumentiert (fr. 282, p. 209f. Deichgräber). Es liegt nahe, daß diese häufig beklagte Philologisierung der Medizin entstanden ist durch Sektenkonkurrenz, die erstens den Aufbau einer Tradition, d.h. eine Kanonisierung und damit Text- und Sinnpflege, zusammen eben Philologie, erforderlich gemacht hat. Die auf diese Weise gesicherte Tradition fungierte als Akkumulation von Autorität gegen potentielle oder reale Gegner und, davon

62 Galen benutzt Archigenes in Loc. aff. in weitem Ausmaß, doch siehe Wellmann (1895: 91): "Gleichwohl hat er uns die Möglichkeit genommen, den Umfang der Benützung des Archigenes aus seinen Citaten nachzuweisen. Er erwähnt ihn in dieser Schrift an elf Stellen, aber nur, um gegen seine Theorieen [sic] zu Felde zu ziehen." Daß er anderswo (Cris. II 8 [IX 670.10$12 \mathrm{Kühn])} \mathrm{Archigenes'} \mathrm{gleichnamige} \mathrm{Schrift} \mathrm{rühmt,} \mathrm{ist} \mathrm{bezeichnend:} \mathrm{dort} \mathrm{ist} \mathrm{dieser} \mathrm{eben} \mathrm{kein}$ klarer Konkurrent.

Z.B. zeigt die kurze Übersicht bei Gossen (1907: 31-33), daß die Geschichte der Pulslehre vollkommen auf den Informationen basiert, die Galen selbst überliefert.

64 Eine gute Beschreibung medizinischer apprenticeship bietet die hippokratische Schrift Eid (dazu Althoff 1993: 215).

Als „decadenza“ verurteilt von Lanza (1972: 426). Kudlien (1974: 197-200) hat gezeigt, daß es sich nicht um einen radikalen Umbruch der Kaiserzeit handelt, sondern da $\beta$ „scholastische", praxisferne Elemente in der alexandrinischen Medizin seit dem Hellenismus vorhanden waren. Zur Philologisierung der Medizin schon im 3. Jh. v. Chr. bei Herophilos siehe Meißner (1999: 169). 
kaum zu trennen, als Werbungbasis für die Rezipienten, um die die Schulen konkurrierten. Zweitens wurde durch den Aufbau dieser Tradition erst Doxographie in weitem Umfang möglich, die - Galen zeigt es - wiederum dem Wettbewerb zugeführt wurde als Ressource für Schulauseinandersetzungen. Diese Phase der Kanonisierung und Selektion, die bis zum sogenannten Alexandrinischen Kanon der Araber die überlieferten medizinischen Werke immer weiter zusammenstrich, ${ }^{66}$ bis sie schließlich zu schieren Begriffsskeletten verkamen (Dietrich 1966: 33; Endreß 1987: 452), lernen wir in der intensiven Polemik Galens einmal gewissermaßen von innen kennen: sie entpuppt sich als Verdrängungswettkampf, der aber eine Fülle literarischer Techniken und Qualitäten als Wettbewerbsmittel ausbildet (ähnlich Swain 1996: 358f.). Die Parallelen zum Evolutionsgeschehen, wie Darwin es sah, sind verführerisch. Die Pulslehre Galens liefert ein Beispiel dafür, wie Wissensgebiete mit fortschreitender Differenzierung die entsprechende Ausdifferenzierung von Literaturformen erzwingen, weil die Fülle des Wissens einzelne Rezeptionsinteressen blockiert und so gerade die Unwahrscheinlichkeit erfolgreicher Wissensvermittlung wieder steigert. Um diese Unwahrscheinlichkeit zu minimieren, entsteht ein Bedürfnis nach differenzierten, gewissermaßen maßgeschneiderten Literaturformen. Das Beispiel der Pulslehre Galens zeigt: Dieses komplexe Gebiet erzeugt eine Einführung (Puls. tir.), eine sechzehn Bücher umfassende Systempragmatie, etliche ausdifferenzierte und dann autonome Metadiskurse (Kommentare zu Herophilos, Archigenes usw.), eine Monographie zu einem Spezialthema (Peri chreias sphygmôn) und schließlich ein Repetitorium (Syn. puls.). Dabei ist die Funktion der systematischen Thesaurierung oder umgekehrt das Bedürfnis nachschlagenden Suchens, das die Systempragmatie nicht befriedigt, noch gar nicht berücksichtigt (teilweise erfüllt durch Definitionssammlungen) ${ }^{67}$ ebensowenig wie die des Vademecums für praktizierende Mediziner (Latours „immutable mobiles" [1990: 26-29] in einem handgreiflicheren Sinn). Solche Texte sind wahrscheinlich in arabischen Galen-Bearbeitungen erhalten. ${ }^{68}$

66 Der alexandrinische Kanon umfaßt nach Hunain ibn Ishâq nur noch sechzehn Schriften, teils ihrerseits Exzerpte (Zusammenfassung in Galen-Übersetzungen 20 [arab. p. 18.19-19.5 / dt. p. 15 Bergsträsser]). Dazu, im Zusammenhang mit „Johannicius“ (= Hunain), Meyerhof (1926: 723f.).

67 Diese sind aber häufig rein doxographisch ausgerichtet: Aristoxenos, Schüler des Alexandros Philalethes (von Staden 1989: 559-563), Herophileer, brachte die Geschichte der Pulslehre in die Form einer Aufeinanderfolge von Definitionen (ähnlich wie Galen seine pneumatischen Vorgänger behandelt in Diff. puls. IV 2-10, der hier vermutlich von Aristoxenos abhängt). Siehe dazu Schöne (1893: bes. 17-24); skeptisch von Staden (1989: 561f.).

Vgl. al-Qiftî über Galen-Exzerpte als Vademecum für den reisenden Arzt: „and making it easy for the reader to memorize them and carry them along in his journey" (Übers. von Gutas 1993: 38). 
Der gesamte literarische Metadiskurs bei Galen läßt sich als Folge der Wettbewerbssituation verstehen: Er muß sich bei seinen Lesern weniger gegen andere Forscher als gegen konkurrierende Fachschriftsteller durchsetzen, weswegen den Fragen der Darstellung und der schriftlichen Wissensvermittlung, der didaskalia, von ihm so viel Aufmerksamkeit gewidmet wird. ${ }^{69}$ So räumt er etwa der Diskreditierung der pneumatischen Lehrmethode, auf der Basis von Definitionen vorzugehen, großen Raum vor allem in Diff. puls. II-IV ein. ${ }^{70}$

Außerdem läßt sich an Galen gut beobachten, wie der Verdrängungskampf der Ärzte schließlich auch für die anhaltende rigorose Prüfung des überlieferten Wissens und seine Selektion sorgt (Meißner 1999: 262): Es gab ja keine spezifisch medizinische Methode, um Hypothesen unter allgemeinem Konsens zu falsifizieren, d.h. Polemik und Suche nach inhärenten Widersprüchen sind das einzige Testverfahren für die Richtigkeit medizinischer Meinungen (abgesehen von der praktischen Ebene des Erfolgs beim Patienten) ${ }^{71}$ und eine notwendige Phase der Kanonisierung von Wissen.

Bei Galen entwickeln sich literarische Ausdrucksformen und sogar Gattungen demnach aus einem bloßen Symptom von Agonalität und Durchsetzungswille zu Mitteln, Dekontextualisierung zu kompensieren und so erst eine sichere Lesekommunikation zu ermöglichen. Der Leser als Objekt des schriftstellerischen Eros (der auch den Fachschriftsteller auszeichnet!) erklärt auch, daß Galen sich selbst weder zu einer medizinischen Sekte bekannte noch selbst eine gründete: die hairesis als Kampfgemeinschaft Gleichgesinnter war als Instrument in Konkurrenzkämpfen inzwischen vom Konzept des medizinischen Massenschriftstellers überholt worden. Dieser wendet die Techniken der Klassikerphilologie nun auf seine eigenen Werke an und strebt damit nach Selbstkanonisierung, dem letzten Schritt zur Monopolisierung des Lesers. Wie vor allem die arabische und lateinische Rezeption zeigen, ist Galen mit seiner anxiety of influence, mit seinem Streben nach überzeitlicher Distinktion, erfolgreich gewesen, gerade in der Pulslehre: seine Rezeptionsgeschichte ist wie im Falle seiner von ihm bewunderten und imitierten Vorgänger Platon und Aristoteles zu einem ,-ismus" geworden ${ }^{72}$ dessen letzte Ausläufer bis ins 19. Jahrhundert reichen. Entsprechend verdrängte er all die anderen griechischen Mediziner, die auf diesem Gebiet arbeiteten und schrieben, so vollständig, daß wir zur Rekon-

Etwa sachlich und theoretisch Praes. puls. I 1 (IX 205.6-206.4 Kühn).

Siehe etwa Diff. puls. IV 2 (VIII 718 Kühn), IV 3 (VIII 720.5-7 Kühn), IV 7 (VIII 734 Kühn), IV 17 (VIII 762f. Kühn: Vergleich zweier didaskaliai, einer definitions- und doxographieintensiven und einer mehr gegenstandsbasierten, d.h. Galens eigener; ferner VIII 763.13-16

71 und VIII 764.10f. Kühn) und Praes. puls. IV 2 (IX 398.2-6 Kühn).

72 Ähnlich Sarton (1954: 31); pace Sarton (1954: 34 Anm. 42).

Die beste Darstellung des „Galenismus“ bei Temkin (1973: 51-192). 
struktion der Geschichte des Pulswissens ${ }^{73}$ praktisch ausschließlich auf die doxographischen Nachrichten Galens und ihre konkurrenzbedingten Verzerrungen angewiesen sind. Erfolgreicher kann die anxiety of influence eines Autors kaum sein.

\section{Literatur}

Textausgaben, Übersetzungen und Kommentare:

Barigazzi, Adelmo (Hrsg.) (1991): Galeno: Sull'ottima maniera d'insegnare / Esortazione alla medicina (Corpus Medicorum Graecorum V 1.1), Berlin.

Bergsträsser, Gotthelf (Hrsg.) (1925): Hunain ibn Ishāq: Über die syrischen und arabischen Galen-Übersetzungen (Abhandlungen für die Kunde des Morgenlandes 17.2), Leipzig (Nachdruck: Nendeln 1966).

De Lacy, Phillip (Hrsg.) (1992): Galen: On Semen (Corpus Medicorum Graecorum V 3.1 ), Berlin.

Iskandar, Albert Z. (Hrsg.) (1988): Galeni De optimo medico cognoscendo (Corpus Medicorum Graecorum Suppl. or. 4), Berlin.

Kühn, Carl Gottlob (Hrsg.) (1821-1833): Claudii Galeni Opera omnia (20 Vol.), Leipzig (Nachdruck: Hildesheim 1965).

Marsden, Eric W. (Hrsg.) (1971): Greek and Roman Artillery. Technical Treatises, Oxford.

Meyerhof, Max \& Joseph Schacht (Hrsg.) (1931): Galen: Über die medizinischen Namen, in: Abhandlungen der preussischen Akademie der Wissenschaften. Philosophisch-historische Klasse Nr. 3/1931, Berlin, 1-43 (1-23 ar., 1-43 dt.).

Müller, Iwan von (Hrsg.) (1891): De propriis libris und De ordine propriorum librorum, in: Ders. (Hrsg.), Claudii Galeni Scripta minora (Vol. 2), Leipzig, 81-124.

Nutton, Vivian (Hrsg.) (1979): Galeni De praecognitione (Corpus Medicorum Graecorum V 8.1), Berlin.

Simon, Max (Hrsg.) (1906): Sieben Bücher Anatomie des Galen, Leipzig.

Sekundärliteratur:

Althoff, Jochen (1999): Aristoteles als Medizindoxograph, in: Philip J. van der Eijk (Hrsg.), Ancient Histories of Medicine, Leiden, 57-94.

Asper, Markus (1998): Zu Struktur und Funktion eisagogischer Texte, in: Wolfgang Kullmann, Jochen Althoff \& Markus Asper (Hrsg.), Gattungen wissenschaftlicher Literatur in der Antike, Tübingen, 309-340.

Asper, Markus (2003): Griechische Lehrtexte. Formen, Funktionen, Differenzierungsgeschichten, Habilitationsschrift Mainz.

Bloom, Harold (1973): The Anxiety of Influence. A Theory of Poetry, Oxford.

73 Ähnlich dominant wirkte die Schriftengruppe um die Methodus medendi: $\mathrm{Zu}$ ars parva und Megategni siehe Kibre \& Kelter (1987: 23). Zur Nachwirkung insgesamt siehe die Übersicht bei Schlange-Schöningen (2003: 10f. Anm. 19). 
Bowersock, Glen W. (1969): Greek Sophists in the Roman Empire, Oxford.

Cowlishaw, L. (1937): Galen, the medical dictator: his life and influence on the progress of medicine, in: Medical Journal of Australia 2, 543-552.

Debru, Armelle (1992): La suffocation hystérique chez Galien et Aétius. Réécriture et emprunt du "je", in: Antonio Garzya (Hrsg.), Tradizione e ecdotica dei testi medici tardoantichi e bizantini, Napoli, 79-89.

Deichgräber, Karl (1957): Galen als Erforscher des menschlichen Pulses. Ein Beitrag zur Selbstdarstellung des Wissenschaftlers (Sitzungsberichte der deutschen Akademie der Wissenschaften zu Berlin. Klasse für Sprache, Literatur \& Kunst 1956/3), Berlin.

Dietrich, Albert (1966): Medicinalia Arabica, Göttingen.

Drabkin, Israel E. (1944): On medical education in Greece and Rome, in: Bulletin of the History of Medicine 15, 333-351.

Endreß, Gerhard (1987): Die wissenschaftliche Literatur, in: Helmut Gätje (Hrsg.), Grundriß der Arabischen Philologie. Vol. 2: Literaturwissenschaft, Wiesbaden, 400-506.

Gossen, Johannes (1907): De Galeni libro qui Synopsis peri sphygmôn inscribitur, Diss. Berlin.

Gutas, Dmitri (1993): Aspects of literary form and genre in Arabic logical works, in: Charles Burnett (Hrsg.), Glosses and Commentaries on Aristotelian Logical Texts. The Syriac, Arabic and Medieval Latin Traditions, London, 29-76.

Hankinson, Robert J. (1994): Galen's concept of scientific progress, in: Aufstieg und Niedergang der römischen Welt II 37.2, 1775-1789.

Hanson, Ann E. (1998): Galen: author and critic, in: Glenn W. Most (Hrsg.), Editing Texts / Texte edieren, Göttingen, 22-53.

Horstmanshoff, Herman F. J. (1995): Galen and his patients, in: Philip J. van der Eijk, Herman F. J. Horstmanshoff \& Pieter H. Schrijvers (Hrsg.), Ancient Medicine in Its Socio-cultural Context (Vol. 1), Amsterdam, 83-99.

Ilberg, Johannes (1974): Über die Schriftstellerei des Klaudios Galenos, Darmstadt [Ursprüngliche Fassung in: Rheinisches Museum für Philologie 44 (1889), 207-239; 47 (1892), 489-514; 51 (1896), 165-196; 52 (1897), 591-623].

Keil, Josef (1905): Ärzteinschrift aus Ephesos, in: Jahreshefte des Österreichischen Archäologischen Institutes in Wien 8, 128-138.

Kibre, Pearl \& Irving A. Kelter (1987): Galen's Methodus medendi in the Middle Ages, in: History \& Philosophy of the Life Sciences 9, 17-36.

Kollesch, Jutta (1979): Ärztliche Ausbildung in der Antike, in: Klio 61, 507-513.

Kudlien, Fridolf (1972): Galen, in: Charles Coulston Gillispie (Hrsg.), Dictionary of Scientific Biography (Vol. 5), 227-237.

Kudlien, Fridolf (1974): Dialektik und Medizin in der Antike, in: Medizinhistorisches Journal 9, 187-201.

Kudlien, Fridolf (1985): „Klassen“-teilung der Ärzte bei Aristoteles, in: Jürgen Wiesner (Hrsg.), Aristoteles: Werk und Wirkung. Paul Moraux gewidmet (Vol. 2), Berlin, 427-435.

Lanza, Diego (1972): „Scientificità“ della lingua e lingua della scienza in Grecia, in: Belfagor 27, 392-429.

Latour, Bruno (1990): Drawing things together, in: Michael Lynch \& Steve Woolgar (Hrsg.), Representation in Scientific Practice, Cambridge, Mass. \& London, 19-68. 
Lloyd, Geoffrey E. R. (1996): Theories and practices of demonstration in Galen, in: Michael Frede \& Gisela Striker (Hrsg.), Rationality in Greek Thought, Oxford, 255 277.

Mansfeld, Jaap (1994): Prolegomena. Questions to Be Settled Before the Study of an Author, or a Text, Leiden.

Meißner, Burkhard (1997): Berufsausbildung in der Antike, in: Max Liedtke (Hrsg.), Berufliche Bildung. Geschichte, Gegenwart, Zukunft, Bad Heilbrunn, 55-99.

Meißner, Burkhard (1999): Die technologische Fachliteratur der Antike. Struktur, Überlieferung und Wirkung technischen Wissens in der Antike (ca. 400 v. Chr. - ca. 500 n. Chr.), Berlin.

Meyerhof, Max (1926): New light on Hunain ibn Ishaq and his period, in: Isis 8, 685724.

Nijhuis, Karin (1995): Greek doctors and Roman patients, in: Philip J. van der Eijk, Herman F. J. Horstmanshoff \& Pieter H. Schrijvers (Hrsg.), Ancient Medicine in Its Socio-cultural Context (Vol. 1), Amsterdam, 49-67.

Nutton, Vivian (1971): Two notes on immunities, in: Journal of Hellenic Studies 61, 5263.

Nutton, Vivian (1984): Galen in the eyes of his contemporaries, in: Bulletin of the History of Medicine 58, 315-324.

Nutton, Vivian (1990): The patient's choice: a new treatise by Galen, in: Classical Quarterly 40, 236-257.

Nutton, Vivian (2002): Ancient medicine: Asclepius transformed, in: Christopher J. Tuplin \& Tracey Elizabeth Rihll (Hrsg.), Science and Mathematics in Ancient Greek Culture, Ox ford, 242-255.

Sarton, George (1954): Galen of Pergamon, Lawrence, Kansas.

Scarborough, John (1981): The Galenic question, in: Sudhoffs Archiv 65, 1-31.

Schlange-Schöningen, Heinrich (2003): Die römische Gesellschaft bei Galen. Biographie und Sozialgeschichte, Berlin \& New York.

Schmitz, Thomas (1997): Bildung und Macht. Zur sozialen und politischen Funktion der zweiten Sophistik in der griechischen Welt der Kaiserzeit, München.

Schöne, Hermann (1893): De Aristoxeni Peri tês Herophilou haireseôs libro tertio decimo a Galeno adhibito, Diss. Bonn 1893.

Sluiter, Ineke (1999): Commentaries and the didactic tradition, in: Glenn W. Most (Hrsg.), Commentaries / Kommentare, Göttingen, 173-205.

Sluiter, Ineke (2000): The dialectics of genre: some aspects of secondary literature and genre in antiquity, in: Mary Depew \& Dirk Obbink (Hrsg.), Matrices of Genre. Authors, Canons, and Society, Cambridge, Mass. \& London, 183-203 und 294-299.

Staden, Heinrich von (1989): Herophilus. The Art of Medicine in Early Alexandria, Cambridge.

Staden, Heinrich von (1994): Author and authority. Celsus and the construction of a scientific self, in: Manuel Enrique Vázquez Buján (Hrsg.), Tradición e innovación de la medicina latina de la antigüedad y de la alta edad media, Santiago de Compostela, 103-117.

Staden, Heinrich von (1997): Galen and the "Second Sophistic", in: Richard Sorabji (Hrsg.), Aristotle and After, London, 33-54.

Staden, Heinrich von (1998): Gattung und Gedächtnis: Galen über Wahrheit und Lehrdichtung, in: Wolfgang Kullmann, Jochen Althoff \& Markus Asper (Hrsg.), Gattungen wissenschaftlicher Literatur in der Antike, Tübingen, 65-94. 
Staden, Heinrich von (1999): Rupture and continuity: Hellenistic reflections on the history of medicine, in: Philip J. van der Eijk (Hrsg.), Ancient Histories of Medicine, Leiden, 143-187.

Strohmaier, Gotthard (1998): Bekannte und unbekannte Zitate in den Zweifeln an Galen des Rhazes, in: Klaus-Dietrich Fischer, Diethard Nickel \& Paul Potter (Hrsg.), Text and Tradition. Studies in Ancient Medicine and Its Transmission Presented to Jutta Kollesch, Leiden, 263-287.

Swain, Simon (1996): Hellenism and Empire. Language, Classicism, and Power in the Greek World AD 50-250, Ox ford.

Temkin, Owsei (1953): Greek medicine as science and craft, in: Isis 44, 213-225.

Temkin, Owsei (1973): Galenism. Rise and Decline of a Medical Philosophy, Ithaca \& London.

van der Eijk, Philip J. (1997): Towards a rhetoric of ancient scientific discourse. Some formal characteristics of Greek medical and philosophical texts (Hippocratic Corpus, Aristotle), in: Egbert J. Bakker (Hrsg.), Grammar as Interpretation. Greek Literature in Its Linguistic Contexts, Leiden, 77-129.

Vegetti, Mario (1994): Enciclopedia e antienciclopedia: Galeno e Sesto Empirico, in: Giuseppe Cambiano, Luciano Canfora \& Diego Lanza (Hrsg.), Lo spazio letterario della Grecia antica. Vol. 1.3: La produzione e la circolazione del testo. I Greci e Roma, Roma, 333-359.

Wellmann, Max (1895): Die pneumatische Schule bis auf Archigenes. In ihrer Entwickelung dargestellt, Berlin.

Wenskus, Otta (1997): Rez. Tamsyn S. Barton, Ancient Astrology (London \& New York 1994) und Tamsyn S. Barton, Power and Knowledge (Ann Arbor 1994), in: Klio 79, 294-295.

Wilamowitz-Moellendorff, Ulrich von (1886): Isyllos von Epidauros, Berlin. 